\title{
Limit Properties of Transition Functions of Continuous-Time Markov Branching Processes
}

\author{
Azam A. Imomov \\ State Testing Center under Cabinet of Ministers of Republic of Uzbekistan, Karshi State University, 17 Kuchabag, \\ 180100 Karshi, Uzbekistan \\ Correspondence should be addressed to Azam A. Imomov; imomov_azam@mail.ru
}

Received 4 July 2014; Revised 7 September 2014; Accepted 21 September 2014; Published 19 October 2014

Academic Editor: Ravi P. Agarwal

Copyright (C) 2014 Azam A. Imomov. This is an open access article distributed under the Creative Commons Attribution License, which permits unrestricted use, distribution, and reproduction in any medium, provided the original work is properly cited.

Consider the Markov Branching Process with continuous time. Our focus is on the limit properties of transition functions of this process. Using differential analogue of the Basic Lemma we prove local limit theorems for all cases and observe invariant properties of considering process.

\section{Introduction and Preliminaries}

Let the random function $Z(t), t \in \mathscr{T}=[0 ;+\infty)$, be the population size at the moment $t$ of monotype individuals that are capable to perish and transform into individuals of random number of the same type. Evolution of individuals occurs by the following scheme. Each individual existing at epoch $t$ independently of his history and of each other for a small time interval $(t ; t+\varepsilon)$ transforms into $j \in \mathbb{N}_{0} \backslash\{1\}$ individuals with probability $a_{j} \varepsilon+o(\varepsilon)$ and with probability $1+a_{1} \varepsilon+o(\varepsilon)$ each individual survives or makes evenly one descendant (as $\varepsilon \rightarrow 0$ ), where $\mathbb{N}_{0}=\{0\} \cup\{\mathbb{N}=1,2, \ldots\}$ and $\left\{a_{j}\right\}$ represent intensities of individuals' transformation where $a_{j} \geq 0$ for $j \in \mathbb{N}_{0} \backslash\{1\}$ and $0<a_{0}<-a_{1}=\sum_{j \in \mathbb{N}_{0} \backslash\{1\}} a_{j}<\infty$. Appearing new individuals undergo transformations under the same way. Aforesaid population process $Z(t)$ describes the branching scheme of population of individuals in which the intensity of transformation is independent of population size and of time. This has been defined first by Kolmogorov and Dmitriev [1] and called the continuous-time Markov Branching Process (MBP).

The process $Z(t)$ is a homogeneous continuous-time Markov chain with the state space on $\mathbb{N}_{0}$. The Markovian nature of this process yields that its transition functions

$$
\begin{aligned}
P_{i j}(t) & :=P_{i}\{Z(t)=j\} \\
& =P\{Z(t+\tau)=j \mid Z(\tau)=i\}, \quad t, \tau \in \mathscr{T},
\end{aligned}
$$

satisfy the Kolmogorov-Chapman equations:

$$
P_{i j}(t)=\sum_{k \in \mathbb{N}} P_{i k}(u) \cdot P_{k j}(t-u), \quad u \leq t,
$$

and a branching property holds for all $i, j \in \mathbb{N}$ :

$$
P_{i j}(t)=\sum_{j_{1}+j_{2}+\cdots+j_{i}=j} P_{1 j_{1}}(t) \cdot P_{1 j_{2}}(t) \cdots P_{1 j_{i}}(t) .
$$

The meaning of equality (3) is the following. If there are $i$ particles at the moment 0 , then their descendants in the moment $t \in \mathscr{T}$ are distributed as the sum of $i$ independent populations, each of which is immediate descendants of single particle; see [2, pp. 148-50].

Thus, for studying of evolution of MBP $Z(t)$ is suffice to set the transition functions $P_{1 j}(t)$. These probabilities in turn, as it has been noted, can be calculated using the local densities $\left\{a_{j}\right\}$ by relation

$$
P_{1 j}(\varepsilon)=\delta_{1 j}+a_{j} \varepsilon+o(\varepsilon), \quad \varepsilon \downarrow 0,
$$

where $\delta_{i j}$ represents Kronecker's delta function. Probability generating functions (GFs) are the main analytical tool in our discussions on MBP. A GF version of the relation (4) is

$$
F(\varepsilon ; s)=s+f(s) \cdot \varepsilon+o(\varepsilon), \quad \varepsilon \downarrow 0,
$$

for all $0 \leq s<1$, where

$$
F(t ; s)=\sum_{j \in \mathbb{N}_{0}} P_{1 j}(t) s^{j}, \quad f(s)=\sum_{j \in \mathbb{N}_{0}} a_{j} s^{j} .
$$


The GF $F(t ; s)$ satisfies functional equation:

$$
F(t+\tau ; s)=F(t ; F(\tau ; s))
$$

with initial condition

$$
F(0 ; s)=s
$$

for any $t, \tau \in \mathscr{T}$. Moreover it satisfies ordinary differential equation

$$
\frac{\partial F(t ; s)}{\partial t}=f(F(t ; s))
$$

and the linear first-order partial differential equation

$$
\frac{\partial F(t ; s)}{\partial t}=f(s) \frac{\partial F(t ; s)}{\partial s}
$$

Equations (9) and (10) correspond to the backward and forward Kolmogorov equations from the general theory of Markov processes with continuous time. It follows from the theory of differential Equations that solutions of these equations are identical; see [3, p. 28]. This solution is unique and satisfies (7) with (8) and is the GF on $s$.

The GF $F(t ; s)$ and all its derivatives

$$
\frac{\partial^{k} F(t ; s)}{\partial s^{k}} \geq 0, \quad k \in \mathbb{N}
$$

are monotone nondecreasing on $0 \leq s<1$. Assuming that

$$
a:=f^{\prime}(1)
$$

is finite entails that $|F(t ; s)| \leq 1$ and $F(t ; 1)=1$; see [3, pp. 27-30].

Using (2) and (3) it is easy to see

$$
F_{i}(t ; s):=\sum_{j \in \mathbb{N}_{0}} P_{i j}(t) s^{j}=[F(t ; s)]^{i}, \quad i \in \mathbb{N} .
$$

Then by (9) and (13), the following can be calculated:

$$
\mathrm{E}_{i} Z(t)=\sum_{j \in \mathbb{N}_{0}} j P_{i j}(t)=i e^{a t} .
$$

The last formula shows that long-term properties of MBP seem variously depending on parameter $a$. Hence the MBP is classified as critical if $a=0$ and subcritical or supercritical if $a<0$ or $a>0$, respectively.

Further we will write everywhere $\mathrm{P}\{*\}$ and $\mathrm{E}[*]$ instead of $\mathrm{P}_{1}\{*\}$ and $\mathrm{E}_{1}[*]$, accordingly.

The event $\{Z(*)=0\}$ is simple absorbing state for any MBP. If $Z(t)=0$ it is said that the process is degenerated at the time $t \in \mathscr{T}$. The probability $P_{i 0}(t)=\mathrm{P}_{i}\{Z(t)=0\}$ denotes the MBP with initial state $Z(0)=i$ is dying out in this time. The extinction probability

$$
q=\lim _{t \rightarrow \infty} P_{10}(t)
$$

can be considered as the probability of all descendants of one initial individual eventually will be lost. Sevast'yanov [4] proved that this probability is the least nonnegative root of $f(s)=0$ and that $q=1$ if the process is nonsupercritical. It directly follows from last results that $q=F(t ; q)$ for any $t \in \mathscr{T}$. Moreover the convergence

$$
\lim _{t \rightarrow \infty} F(t ; s)=q
$$

holds uniformly for all $0 \leq s \leq r<1$. Owing to the last assertion the function $R(t ; s)=q-F(t ; s)$ plays a very important role in observing limit behaviors of MBP.

In the case of $a \leq 0$

$$
R(t):=R(t ; 0)=1-P_{10}(t)=\mathrm{P}\{Z(t)>0\}
$$

is a surviving probability of posterities of the single particle at the time $t$. An asymptote of this probability has first been established by Sevastyanov [4]. Refinement of his results is obtained in [5-7].

In supercritical situation

$$
R(t)=q-F(t ; 0) \equiv \mathrm{P}\{t<T<\infty\},
$$

where $q<1$ and $T=\inf \{t \in \mathscr{T}: Z(t)=0\}$. This variable denotes an extinction time of MBP. That is

$$
\{T=t\} \Longleftrightarrow\{Z(\tau)>0, \tau<t, Z(t)=0\} .
$$

Note that if $a \leq 0$ then $\mathrm{P}\{Z(t)>0\} \equiv \mathrm{P}\{T>t\}$ and

$$
0 \leq P_{1 j}(t) \leq \mathrm{P}\{T>t\} \longrightarrow 0, \quad t \longrightarrow \infty .
$$

So long-term properties of process $Z(t)$ are investigated on non-zero trajectories that is conditioned to event $T>t$. In this context we state some classical results on asymptote of conditioned distribution $\mathrm{P}\{*\}=\mathrm{P}\{* \mid T>t\}$ derived first by Chistyakov [8] and Sevastyanov [4].

Theorem A (see [4]). If $a<0$ and $\sum_{j \in \mathbb{N}} a_{j} j \ln j<\infty$, then

$$
\frac{P_{1 j}(t)}{\mathrm{P}\{T>t\}}=\mathrm{P}\{Z(t)=j \mid Z(t)>0\} \longrightarrow P_{j}^{*}, \quad t \longrightarrow \infty,
$$

where the corresponding GF

$$
\sum_{j \in \mathbb{N}} P_{j}^{*} s^{j}=1-\exp \left\{a \int_{0}^{s} \frac{d x}{f(x)}\right\} .
$$

Theorem B (see [4]). If $a=0$ and $2 b:=f^{\prime \prime}(1)<\infty$, then

$$
\lim _{t \rightarrow \infty} \mathrm{P}\left\{\frac{Z(t)}{b t}<u \mid Z(t)>0\right\}=1-e^{-u}, \quad u>0 .
$$

Theorem C (see [8]). If $a=0, f^{(4)}(1)<\infty$, and $j / b t$ is bounded, then

$$
\frac{P_{1 j}(t)}{\mathrm{P}\{T>t\}}=\frac{1}{b t} \exp \left\{-\frac{j}{b t}\right\}+r(t), \quad t \longrightarrow \infty,
$$

where $r(t)=O\left(\sqrt{\ln t} / \sqrt{t^{3}}\right)$. 
In supercritical case the following theorem holds.

Theorem D (see [4]). If $a>0$ and $2 b:=f^{\prime \prime}(1)<\infty$, then $Z(t) / e^{a t}$ converges as $t \rightarrow \infty$ in the mean square sense and with probability one to a random variable, having continuous distribution with finite variance.

In Section 2 we take assertions about asymptotical decay of the function $R(t ; s)$ for all cases as the Basic Lemma of the theory of MBP. Afterwards, the differential analogue of the Basic Lemma will be established. Hereupon we will find out local limit Theorems 4-6.

In Section 3 we observe invariance properties of states of MBP. We start this section with the proof of Lemma 7 on monotone ratio limit property of $P_{i j}(t) / P_{11}(t)$. We discuss the role of these limits as invariant measure for $Z(t)$ and will find out a corresponding limit GF. And also, we take complete accounts on the asymptotic properties of transition functions using Lemma 7 (Theorems 13 and 14). In noncritical case (Theorem 13) we obtain an exponential invariance property of MBP $Z(t)$ and discuss the criteria for the $\lambda_{Z}$-classification of its state space, where $\lambda_{Z}$ is the decay parameter. Herewith we will follow results of monograph [9, chapter 6$]$ and the paper [10]. In critical situation Theorem 14 improves the result of Theorem $C$. Moreover we will establish that there is a $\operatorname{MBP} \widetilde{Z}(t)$ with transition function $\mathrm{P}_{i}\{Z(t)=j \mid t<T<\infty\}$ which in case $a \neq 0$ has ergodicity property. Finally we refine Theorems A and D consolidating them in the Theorem 16 under minimal moment conditions.

\section{The Basic Lemma and Its Differential Analogue}

As it already was noted in the previous section, asymptotic properties of our MBP are regulated in essence by parameter $a$. In the case $a>1$ the extinction probability $q<1$. And if $a \leq 1$ then the process $Z(t)$ eventually degenerates. Thus the main analytical tool is GF $F(t ; s)$ of distributions of the population size of MBP for which (7)-(10) hold.

First we observe an asymptotic expansion of the function $R(t ; s)$. An assertion describing this expansion, due to its importance, is called the Basic Lemma of the theory of MBP (in fact this name is usually used for the critical case). Let's consider the noncritical case. Multiplying by $f^{\prime}(q) \cdot(F(t ; s)-q)$ the both sides of (9) yields

$$
\begin{aligned}
& \frac{d F(t ; s)}{F(t ; s)-q}-\frac{f(F(t ; s))-f^{\prime}(q) \cdot(F(t ; s)-q)}{f(F(t ; s)) \cdot(F(t ; s)-q)} d F(t ; s) \\
& \quad=f^{\prime}(q) d t .
\end{aligned}
$$

After integration on $[0 ; t] \subset \mathscr{T}$ it receives from this equality that

$$
\begin{aligned}
& \ln \frac{R(t ; s)}{R(0 ; s)} \\
& \quad=f^{\prime}(q) t+\int_{0}^{t} \frac{f(F(t ; s))-f^{\prime}(q) \cdot(F(t ; s)-q)}{f(F(t ; s)) \cdot(F(t ; s)-q)} d F(t ; s) .
\end{aligned}
$$

Changing of variables it follows from here

$$
\frac{R(t ; s)}{R(0 ; s)}=\beta^{t} \cdot \exp \left\{\int_{s}^{F(t ; s)}\left[\frac{1}{u-q}-\frac{f^{\prime}(q)}{f(u)}\right] d u\right\} .
$$

where $\beta:=\exp \left\{f^{\prime}(q)\right\}$. Considering that $\sup _{0 \leq s<1} F(t ; s) \rightarrow$ $q$, in integral of the right side of (27), it is possible to go to the limit as $t \rightarrow \infty$. Then we directly would receive asymptote of the function $R(t ; s)$ in the case of $a \neq 0$. In the critical situation the asymptotic representation for $R(t ; s)$ was established first by Sevastyanov [4] which is given in the formula (30) below. Aggregating these facts we can formulate the following fundamental importance statement.

Lemma 1 (Basic Lemma). The following assertions are true.

(i) If $a \neq 0$, then

$$
R(t ; s)=\mathscr{A}(s) \cdot \beta^{t}(1+o(1)), \quad t \longrightarrow \infty ;
$$

here $\beta:=\exp \left\{f^{\prime}(q)\right\}$ and

$$
\mathscr{A}(s)=(q-s) \exp \left\{\int_{s}^{q}\left[\frac{1}{u-q}-\frac{f^{\prime}(q)}{f(u)}\right] d u\right\} .
$$

(ii) Let $a=0$. If the second moment $f^{\prime \prime}(1)=: 2 b$ is finite, then

$$
R(t ; s)=\frac{1-s}{b t(1-s)+1}(1+o(1)), \quad t \longrightarrow \infty .
$$

Now we are interested in asymptotic properties of the function $\partial R(t ; s) / \partial s$. The following assertions describe limit behaviors of this function and we refer to that as the differential analogue (DA) of the Basic Lemma. In the paper [11] this lemma showed without supercritical situation.

Lemma 2 (DA Basic Lemma). The following assertions are true.

(i) Let $a \neq 0$. Then

$$
\frac{\partial R(t ; s)}{\partial s}=\frac{f^{\prime}(q)}{f(s)} \mathscr{A}(s) \cdot \beta^{t}(1+o(1)), \quad t \longrightarrow \infty,
$$

where the function $\mathscr{A}(s)$ is defined in (28).

(ii) Let $a=0$. If the second moment $f^{\prime \prime}(1)=: 2 b$ is finite, then

$$
\frac{\partial R(t ; s)}{\partial s}=\frac{-b(1-s)^{2}}{f(s)[b t(1-s)+1]^{2}}(1+o(1)), \quad t \longrightarrow \infty .
$$

Proof. Equating left-hand sides of (9) and (10) yields the following identity:

$$
\frac{\partial R(t ; s)}{\partial s}=-\frac{f(1-R(t ; s))}{f(s)} .
$$


In the case $a<0$ considering the relation $f(s)=a(s-1)(1+$ $o(1))$ which holds as $s \uparrow 1$ and seeing that $R(t ; s) \rightarrow 0$ as $t \rightarrow \infty$ we obtain

$$
f(1-R(t ; s))=-a R(t ; s)(1+o(1)) .
$$

Hence it follows

$$
\frac{\partial R(t ; s)}{\partial s}=\frac{a}{f(s)} R(t ; s)(1+o(1)), \quad t \longrightarrow \infty .
$$

Similarly when $a>0$ we see $f(s) \sim f^{\prime}(q)(s-q)$ as $s \rightarrow q$. Therefore

$$
\frac{\partial R(t ; s)}{\partial s}=\frac{f^{\prime}(q)}{f(s)} R(t ; s)(1+o(1)), \quad t \longrightarrow \infty
$$

for all $0 \leq s<1$. Using (28) in (35) and (35*) we will get (31).

In the case $a=0$ by the same way and seeing $b<\infty$ we receive

$$
\frac{\partial R(t ; s)}{\partial s}=-\frac{b}{f(s)} R^{2}(t ; s)(1+o(1)), \quad t \longrightarrow \infty
$$

Now assertion (32) follows from relations (30) and (36).

Last statements show that in research of the noncritical case our discussion will depend on properties of the function $\mathscr{A}(s)$. Thereby we have to observe properties of this function. The following lemma describes basic properties of this function.

Lemma 3. The function $\mathscr{A}(s)$ is defined on $0 \leq s<1$ that is continuously, monotone decreasing, and concave everywhere. Moreover if $a>0$ or $a<0$ and

$$
\sum_{j \in \mathbb{N}} a_{j} j \ln j<\infty
$$

then $0<\mathscr{A}(0)<\infty, \mathscr{A}(q)=0, \mathscr{A}^{\prime}(q)=-1$, and

$$
\lim _{s \uparrow 1} \mathscr{A}(s)= \begin{cases}0, & a<0, \\ -\infty, & a>0 .\end{cases}
$$

$\mathscr{A}(s)$ is the solution of Schroeder equation:

$$
\mathscr{A}(F(t ; s))=\beta^{t} \cdot \mathscr{A}(s) .
$$

On the set of $0 \leq s<q$ this solution is unique.

Proof. In fact the function $\mathscr{A}(s)$ is defined on the set of $0 \leq s<$ 1 , since that is outcome from (27) as $t \rightarrow \infty$. Its continuity is obvious. From (29) we have

$$
\mathscr{A}^{\prime}(s)=\frac{f^{\prime}(q)}{f(s)} \mathscr{A}(s) \text {. }
$$

It is known that the GF $f(s)$ is convex everywhere. For $0 \leq s<$ $q$ it is strictly positive and monotone decreasing. As $\mathscr{A}(s)>$ 0 and $f^{\prime}(q)<0$ it follows $\mathscr{A}^{\prime}(s)<0$. Hence the function $\mathscr{A}(s)$ is monotone decreasing. By the same reasoning we will be convinced that $\mathscr{A}(s)$ is monotone decreasing for $q \leq s<1$.

We know that in point $s=q$ the GF $f(s)$ changes its sign from plus to minus and its derivative $f^{\prime}(s)$ monotonously increases. Therefore considering that $\mathscr{A}^{\prime}(s)<0$ we find out that

$$
\mathscr{A}^{\prime \prime}(s)=\frac{f^{\prime}(q)-f^{\prime}(s)}{f(s)} \cdot \mathscr{A}^{\prime}(s)<0 .
$$

This implies the concavity of $\mathscr{A}(s)$.

In the case $a<0$ the condition (37) holds if only if when

$$
\int_{0}^{1} \frac{f(u)-a \cdot(u-1)}{(u-1) f(u)} d u=\ln \mathscr{A}(0)<\infty
$$

see [3, p. 57]. One can see when $a<0$, the condition (42) entails $\mathscr{A}(0)>0$ and this is finite. In the case of $a>0$ it can easily be convinced that $0<\mathscr{A}(0)<\infty$. The assertion $\mathscr{A}(q)=0$ directly follows from (42) in the case $a<0$. If $a>0$, then the integrand in (29) stays bounded as $s \rightarrow q$ and hence $\mathscr{A}(q)=0$. Considering $f(s) \sim f^{\prime}(q)(s-q)$ as $s \rightarrow q$, and that integrand is bounded as $u \rightarrow q$, entails from (29) that

$$
\mathscr{A}^{\prime}(q)=\lim _{s \rightarrow q} \mathscr{A}^{\prime}(s)=\lim _{s \rightarrow q} \frac{f^{\prime}(q)}{f(s)} \mathscr{A}(s)=\lim _{s \rightarrow q} \frac{\mathscr{A}(s)}{s-q}=-1 .
$$

In the case $a>0$ we see that integrand in (29) increases to $-\infty$ as $s \uparrow 1$. Therefore,

$$
\mathscr{A}(s \uparrow 1)=-\infty
$$

Now designating $K(u)$ the integrand in (29) we see that function $\mathscr{A}(s)$ actually satisfies (39):

$$
\begin{aligned}
\beta^{t} \mathscr{A}(s)= & (q-s) \beta^{t} \exp \left\{\int_{s}^{F(t ; s)} K(u) d u\right\} \\
& \cdot \exp \left\{\int_{F(t ; s)}^{q} K(u) d u\right\} \\
= & (q-F(t ; s)) \exp \left\{\int_{F(t ; s)}^{q} K(u) d u\right\} \\
= & \mathscr{A}(F(t ; s)) .
\end{aligned}
$$

The equality (27) was used in the last step.

To observe the uniqueness of the solution of (39), we follow the method from monograph $[12, \mathrm{p} .14]$. Suppose $\widetilde{\mathscr{A}}(s)$ is an arbitrary solution of (39). Then it as well as $\mathscr{A}(s)$ satisfies the equation

$$
\mathscr{A}^{\prime}(F(t ; s)) \cdot F^{\prime}(t ; s)=\beta^{t} \cdot \mathscr{A}^{\prime}(s) \text {. }
$$


Hereinafter, if not otherwise stated, the derivative symbol for the function $F(t ; s)$ should be understood by $s$. It follows from (46)

$$
\frac{\mathscr{A}^{\prime}(s)}{\widetilde{\mathscr{A}}^{\prime}(s)}=\frac{\mathscr{A}^{\prime}(F(t ; s))}{\widetilde{\mathscr{A}}^{\prime}(F(t ; s))}
$$

We have already proved that the solution of (39) is concave; hence both $\mathscr{A}^{\prime}(s)$ and $\widetilde{\mathscr{A}}^{\prime}(s)$ are monotone decrease. Since $F(t ; 0) \uparrow q$ for all $0 \leq s<q$, there always exists some $\tau \in \mathscr{T}$ and some arbitrary small $\varepsilon \in \mathscr{T}$ such that $F(\tau ; 0) \leq s \leq$ $F(\tau+\varepsilon ; 0)$. Then by combining the equalities (46) and (47) we can write following relations:

$$
\begin{aligned}
\frac{\mathscr{A}^{\prime}(s)}{\widetilde{\mathscr{A}}^{\prime}(s)} & \leq \frac{\mathscr{A}^{\prime}(F(t ; F(\tau ; 0)))}{\widetilde{\mathscr{A}}^{\prime}(F(t ; F(\tau+\varepsilon ; 0)))} \\
& \leq \frac{\mathscr{A}^{\prime}(F(t+\tau ; 0))}{\widetilde{\mathscr{A}}^{\prime}(F(t+\tau ; 0))} \cdot \frac{\widetilde{\mathscr{A}^{\prime}}(F(t+\tau ; 0))}{\widetilde{\mathscr{A}}^{\prime}(F(t+\tau+\varepsilon ; 0))} \\
& \leq \frac{\mathscr{A}^{\prime}(0)}{\widetilde{\mathscr{A}}^{\prime}(0)} \cdot \frac{F^{\prime}(t+\tau+\varepsilon ; 0)}{F^{\prime}(t+\tau ; 0) \cdot \beta^{\varepsilon}} \\
& \leq \frac{\mathscr{A}^{\prime}(0)}{\widetilde{\mathscr{A}}^{\prime}(0)} \cdot \frac{F^{\prime}(\varepsilon ; F(t+\tau ; 0))}{\beta^{\varepsilon}} .
\end{aligned}
$$

Since $F(t ; 0) \uparrow q$, we see that $F^{\prime}(\varepsilon ; F(t ; 0)) \uparrow \beta^{\varepsilon}$ as $t \rightarrow \infty$. Denoting $E(t):=F^{\prime}(t ; q)$ and using (9) yield

$$
\frac{d E(t)}{d t}=f^{\prime}(q) E(t)
$$

Thus solving this equation with $F(0 ; s)=s$ it follows $F^{\prime}(t ; q)=$ $\beta^{t}$. So taking limit as $t \rightarrow \infty$ in the inequality (48), we get

$$
\frac{\mathscr{A}^{\prime}(s)}{\widetilde{\mathscr{A}}^{\prime}(s)} \leq \frac{\mathscr{A}^{\prime}(0)}{\widetilde{\mathscr{A}}^{\prime}(0)} \text {. }
$$

A similar reasoning implies a converse inequality. Thus we receive

$$
\frac{\mathscr{A}^{\prime}(s)}{\widetilde{\mathscr{A}}^{\prime}(s)}=\frac{\mathscr{A}^{\prime}(0)}{\widetilde{\mathscr{A}}^{\prime}(0)}=\text { const. }
$$

As $\mathscr{A}(0)=\widetilde{\mathscr{A}}(0)$, then it follows from (51) that $\mathscr{A}(s)=\widetilde{\mathscr{A}}(s)$.

Lemma 3 is proved completely.

Let us return to DA Basic Lemma. One can see it has simple appearance, but as it will be visible further, this lemma represents fundamental importance in our discussions. Namely, it will easily be calculated that

$$
\left.\frac{\partial F(t ; s)}{\partial s}\right|_{s=0}=P_{11}(t)
$$

is the probability of return of the process with initial state $Z(0)=1$ to the one in time $t$. In fact $f(0)=a_{0}>0$, so putting $s=0$ in (31) and (32), we will receive directly consequences from the DA Basic Lemma as local limit theorems below.
Theorem 4. Let $a<0$. If integral (42) is finite then

$$
e^{|a| t} P_{11}(t)=\frac{|a|}{a_{0}} \mathscr{A}(0)(1+o(1)), \quad t \longrightarrow \infty .
$$

Theorem 5. Let $a>0$. Then

$$
\beta^{-t} P_{11}(t)=\frac{\left|f^{\prime}(q)\right|}{a_{0}} \mathscr{A}(0)(1+o(1)), \quad t \longrightarrow \infty .
$$

Theorem 6. Let $a=0$. If the second moment $f^{\prime \prime}(1)=: 2 b$ is finite then

$$
t^{2} P_{11}(t)=\frac{1}{a_{0} b}\left(1+O\left(\frac{1}{t}\right)\right), \quad t \longrightarrow \infty .
$$

These theorems demonstrate a "rate of irrevocability" of the initial state $\{Z(0)=1\}$. In turn they will play a key role in studying of asymptotic properties of transition function $P_{i j}(t)$ for any $i$ and $j$. Designating $R_{i}(t ; s)=q^{i}-F_{i}(t ; s)$, it follows that

$$
\frac{\partial R_{i}(t ; s)}{\partial s}=i F^{i-1}(t ; s) \frac{\partial R(t ; s)}{\partial s}
$$

Since $F_{i}(t ; s)=[F(t ; s)]^{i} \rightarrow q^{i}$, we see from here that

$$
P_{i 1}(t)=i q^{i-1} P_{11}(t)
$$

Hence using assertions (53)-(55) we can receive probabilities of return of process $Z(t)$ from any state $i$ to initial one.

In the basis of all our further results which will appear in the next section are consequences of the DA Basic Lemma set forth above.

\section{Ergodic Behavior of Transition Functions}

Continuing researches of the asymptote of transition functions $P_{i j}(t)$ we deal with problems of ergodicity and existence of invariant measure for MBP. Ergodicity properties of arbitrary continuous-time Markov chains are described in the monograph of Anderson [9, Chapter 6]. We see below that when $a \neq 0$ the chain $Z(t)$ has an exponential invariant behavior.

An invariant (or stationary) measure of the MBP with the transition function $\left\{P_{i j}(t)\right\}$ is a set of nonnegative numbers $\left\{v_{j}, j \in \mathbb{N}_{0}\right\}$ satisfying equation

$$
v_{j}=\sum_{k \in \mathbb{N}_{0}} v_{k} P_{k j}(t)
$$

In this case the property (58) determines an invariant property of the measure $\left\{v_{j}\right\}$ concerning transition functions $\left\{P_{i j}(t)\right\}$. If $\sum_{j \in \mathbb{N}_{0}} v_{j}<\infty$ (or without loss of generality $\left.\sum_{j \in \mathbb{N}_{0}} v_{j}=1\right)$ then it is called as invariant distribution.

The following lemma, about monotone ratio limit property of transition functions, plays an important role along with the Lemma 2 in our purpose. 
Lemma 7 (monotone ratio). For all $j \in \mathbb{N}$

$$
\frac{P_{i j}(t)}{P_{11}(t)} \uparrow i q^{i-1} \mu_{j}<\infty, \quad t \longrightarrow \infty,
$$

where $\mu_{j}=\lim _{t \rightarrow \infty} P_{1 j}(t) / P_{11}(t)$.

Proof. Formally differentiation of (7) implies the following equality:

$$
F^{(j)}(t+\tau ; s)=D_{j}(\tau, t ; s)+F^{\prime}(\tau ; F(t ; s)) F^{(j)}(t ; s),
$$

where the top index means derivatives of the corresponding order by $s$ and expression $D_{j}(\tau, t ; s)$ represents the power series with nonnegative coefficients. In our case $P_{11}(t)>0$ for any $t \in \mathscr{T}$. Accordingly by means of equality (60) follows

$$
\begin{aligned}
\frac{P_{1 j}(t+\tau)}{P_{11}(t+\tau)} & =\frac{1}{j !} \frac{F^{(j)}(t+\tau ; 0)}{F^{\prime}(t+\tau ; 0)} \\
& =\frac{1}{j !} \frac{D_{j}(\tau, t ; 0)+F^{\prime}(\tau ; F(t ; 0)) F^{(j)}(t ; 0)}{F^{\prime}(\tau ; F(t ; 0)) F^{\prime}(t ; 0)} \\
& \geq \frac{1}{j !} \frac{F^{(j)}(t ; 0)}{F^{\prime}(t ; 0)}=\frac{P_{1 j}(t)}{P_{11}(t)} .
\end{aligned}
$$

Therefore the ratio $P_{1 j}(t) / P_{11}(t)$ converges increasing to a finite positive limit as $t \rightarrow \infty$ that we denote

$$
\mu_{j}=\lim _{t \rightarrow \infty} \frac{P_{1 j}(t)}{P_{11}(t)} .
$$

Now consider $P_{i j}(t) / P_{11}(t)$. Letting

$$
\mathscr{M}_{i}(t ; s)=\sum_{j \in \mathbb{N}} \frac{P_{i j}(t)}{P_{11}(t)} s^{j}, \quad i \in \mathbb{N} .
$$

for all $0 \leq s<1$, we can write a following chain of equalities:

$$
\begin{aligned}
\mathscr{M}_{i}(t ; s) & =\sum_{j \in \mathbb{N}_{0}} \frac{P_{i j}(t)}{P_{11}(t)} s^{j}-\frac{P_{i 0}(t)}{P_{11}(t)}=\frac{F^{i}(t ; s)-F^{i}(t ; 0)}{P_{11}(t)} \\
& =\frac{F(t ; s)-F(t ; 0)}{P_{11}(t)} \cdot \sum_{m=1}^{i} F^{i-m}(t ; s) F^{m-1}(t ; 0) \\
& =\sum_{m=1}^{i} F^{i-m}(t ; s) F^{m-1}(t ; 0) \cdot \sum_{j \in \mathbb{N}} \frac{P_{1 j}(t)}{P_{11}(t) s^{j}} .
\end{aligned}
$$

Owing to (62) and convergence $F(t ; s) \rightarrow q$ uniformly for all $0 \leq s<1$ as $t \rightarrow \infty$, it follows from last equality that

$$
\mathscr{M}_{i}(t ; s) \longrightarrow i q^{i-1} \cdot \sum_{j \in \mathbb{N}} \mu_{j} s^{j}
$$

Hence by the continuity theorem for GF we get (59).

\section{Denoting}

$$
\mathscr{M}(s)=\sum_{j \in \mathbb{N}} \mu_{j} s^{j},
$$

in concordance with Lemma 7

$$
\mathscr{M}_{i}(t ; s) \sim i q^{i-1} \mathscr{M}(t ; s) \longrightarrow i q^{i-1} \cdot \mathscr{M}(s), \quad t \longrightarrow \infty,
$$

where $\mathscr{M}(t ; s):=\mathscr{M}_{1}(t ; s)$. Within our purpose we will be interested in properties of $\left\{\mu_{j}, j \in \mathbb{N}\right\}$. In view of nonnegativeness of these numbers a limit function

$$
\mathscr{M}(s)=\lim _{t \rightarrow \infty} \mathscr{M}(t ; s)
$$

is monotone nondecreasing with $s$. In concordance with the relation (67), for the analysis of the limit of $P_{i j}(t) / P_{11}(t)$ it suffices to consider the function $\mathscr{M}(t ; s)$.

The following theorem holds.

Theorem 8. Nonnegative numbers $\left\{\mu_{j}\right\}$ satisfy invariant equation

$$
\beta^{t} \cdot \mu_{j}=\sum_{k \in \mathbb{N}} \mu_{k} P_{k j}(t), \quad j \in \mathbb{N} .
$$

The function $\mathscr{M}(s)$ satisfies the functional equation

$$
\beta^{t} \cdot \mathscr{M}(s)=\mathscr{M}(F(t ; s))-\mathscr{M}(F(t ; 0)),
$$

and that converges for $0 \leq s<1$.

Proof. Differentiating of (7) gives

$$
P_{11}(t+\tau)=F^{\prime}(t+\tau ; 0)=F^{\prime}(\tau ; F(t ; 0)) \cdot P_{11}(t),
$$

for any $t, \tau \in \mathscr{T}$. Since $F^{\prime}(\tau ; F(t ; 0)) \uparrow \beta^{\tau}$ it follows from last equality that

$$
\frac{P_{11}(t+\tau)}{P_{11}(t)} \uparrow \beta^{\tau}, \quad t \rightarrow \infty .
$$

Now using the Kolmogorov-Chapman equation (2),

$$
P_{1 j}(t+\tau)=\sum_{k \in \mathbb{N}} P_{1 k}(t) \cdot P_{k j}(\tau),
$$

we have

$$
\frac{P_{1 j}(t+\tau)}{P_{11}(t+\tau)} \cdot \frac{P_{11}(t+\tau)}{P_{11}(t)}=\sum_{k \in \mathbb{N}} \frac{P_{1 k}(t)}{P_{11}(t)} \cdot P_{k j}(\tau) .
$$

Taking limit here as $t \rightarrow \infty$ and seeing (62) and (72) we attain (69). Transforming to GF in both sides of (69) and taking into account (13), we find

$$
\begin{aligned}
\beta^{t} \sum_{j \in \mathbb{N}} \mu_{j} s^{j} & =\sum_{j \in \mathbb{N}} \sum_{k \in \mathbb{N}} \mu_{k} P_{k j}(t) s^{j} \\
& =\sum_{k \in \mathbb{N}} \mu_{k}\left[\sum_{j \in \mathbb{N}_{0}} P_{k j}(t) s^{j}-P_{k 0}(t)\right] \\
& =\sum_{k \in \mathbb{N}} \mu_{k}(F(t ; s))^{k}-\sum_{k \in \mathbb{N}} \mu_{k}(F(t ; 0))^{k} .
\end{aligned}
$$

Obtained equality is equivalent to (70). 
Let us prove now convergence of function $\mathscr{M}(s)$. Put

$$
S=\left\{j \in \mathbb{N}: P_{1 j}(t)>0, t \in \mathscr{T}\right\} .
$$

Due to $P_{11}(t)>0$ and in accordance with (62) all $\mu_{j}<\infty, j \in$ $\mathbb{N}$ and $\mu_{j}>0$ for $j \in \mathrm{S}$. Moreover $\mu_{1}=1$. Then considering the branching property (3) for any fixed $t=\tau_{0}$ we have from (69) that

$$
\begin{aligned}
\beta^{\tau_{0}} & =\beta^{\tau_{0}} \mu_{1}=\sum_{i \in \mathbb{N}} \mu_{i} P_{i 1}\left(\tau_{0}\right) \\
& =\sum_{i \in \mathbb{N}} \mu_{i} i P_{10}^{i-1}\left(\tau_{0}\right) P_{11}\left(\tau_{0}\right) \\
& =\frac{P_{11}\left(\tau_{0}\right)}{P_{10}\left(\tau_{0}\right)} \sum_{i \in \mathbb{N}} i \mu_{i} P_{10}^{i}\left(\tau_{0}\right) \\
& >\frac{P_{11}\left(\tau_{0}\right)}{P_{10}\left(\tau_{0}\right)} \sum_{i \in \mathbb{N}} \mu_{i} P_{10}^{i}\left(\tau_{0}\right)=\frac{P_{11}\left(\tau_{0}\right)}{P_{10}\left(\tau_{0}\right)} \mathscr{M}\left(F\left(\tau_{0} ; 0\right)\right) .
\end{aligned}
$$

From last equality it follows $\mathscr{M}(F(t ; 0))<\infty$ for all $t \in \mathscr{T}$. That in turn implies $\mathscr{M}(s)<\infty$ for $0 \leq s<q$ since $F(t ; 0) \uparrow$ $q$. Here we have considered also a monotone property of the function $\mathscr{M}(s)$. So in the case $a \leq 0$ a convergence of $\mathscr{M}(s)$ is proved because $q=1$. Now prove the convergence of this function for $s \in[q ; 1)$ in the case $a>0$. According to the monotone ratio Lemma 7

$$
\begin{aligned}
\mathscr{M}(s) & =\lim _{t \rightarrow \infty} \mathscr{M}(t ; s)=\lim _{t \rightarrow \infty} \sum_{j \in \mathbb{N}} \frac{P_{1 j}(t)}{P_{11}(t)} s^{j} \\
& =\lim _{t \rightarrow \infty} \frac{F(t ; s)-F(t ; 0)}{P_{11}(t)} \leq \lim _{t \rightarrow \infty} \frac{F^{\prime}(t ; s)}{P_{11}(t)} .
\end{aligned}
$$

On the other hand it follows from (31) and (54) that

$$
\lim _{t \rightarrow \infty} \frac{F^{\prime}(t ; s)}{P_{11}(t)}=\frac{a_{0}}{f(s)} \frac{\mathscr{A}(s)}{\mathscr{A}(0)},
$$

where the function $\mathscr{A}(s)$ is defined in (29). In Lemma 3 , it is proved that this function is finite for $0 \leq s<1$ and $\mathscr{A}(0)>0$. Hence $\mathscr{A}(s) / \mathscr{A}(0)<\infty$ and this implies the convergence of $\mathscr{M}(s)$.

Theorem 9. Equation (70) has unique solution for $0 \leq s<q$ that is power series with nonnegative coefficients.

Proof. As already proved in Lemma 3 the function $\mathscr{A}(s)$ is the solution of Schroeder equation

$$
\mathscr{A}(F(t ; s))=\beta^{t} \cdot \mathscr{A}(s)
$$

and that is unique. Putting $\widehat{\mathscr{A}}(s)=\mathscr{A}(s)-\mathscr{A}(0)$ from here we take

$$
\beta^{t} \widehat{\mathscr{A}}(s)=\widehat{\mathscr{A}}(F(t ; s))-\widehat{\mathscr{A}}(F(t ; 0)) .
$$

The last equation is equivalent to (70) that in turn in concordance with our designation has a unique solution.
In subsequent theorems a form of the limit function $\mathscr{M}(s)=\lim _{t \rightarrow \infty} \mathscr{M}(t ; s)$ will be obtained.

Theorem 10. If $a<0$ and $\sum_{j \in \mathbb{N}} a_{j} j \ln j<\infty$ or $a>0$, then

$$
\mathscr{M}(s)=\frac{a_{0}}{\left|f^{\prime}(q)\right|} \cdot\left[1-\frac{\mathscr{A}(s)}{\mathscr{A}(0)}\right] .
$$

Theorem 11. Let $a=0$. If the second moment $f^{\prime \prime}(1)=: 2 b$ is finite, then

$$
\mathscr{M}(t ; s)=\frac{a_{0}}{b} \cdot \frac{s}{1-s}+\alpha(t ; s),
$$

where $\sup _{0 \leq s<1}|\alpha(t ; s)|=O(1 / t)$ as $t \rightarrow \infty$.

Proofs of last theorems come out from Lemma 1 and Theorems $4-6$ by following. Recall $R(t ; s)=q-F(t ; s)$ and we write

$$
\mathscr{M}(t ; s)=\frac{F(t ; s)-F(t ; 0)}{P_{11}(t)}=\left(1-\frac{R(t ; s)}{R(t)}\right) \cdot \frac{R(t)}{P_{11}(t)},
$$

where $R(t):=R(t ; 0)$.

As noted in the proof of the Lemma 3, the condition of Theorem 10 in the case $a<0$ is equivalent to that the integral

$$
\int_{0}^{1} \frac{f(u)-a \cdot(u-1)}{(u-1) f(u)} d u=\ln \mathscr{A}(0)
$$

is finite. Therefore from (28)

$$
\lim _{t \rightarrow \infty} \frac{R(t ; s)}{R(t)}=\frac{\mathscr{A}(s)}{\mathscr{A}(0)} .
$$

On the other hand from (28) and (53) $R(t) / P_{11}(t) \rightarrow a_{0} /|a|$ as $t \rightarrow \infty$. Combining this fact and equalities (84) and (86) yields (82).

In the case of $a>0$ by the same way from (84) and (86) and that $R(t) / P_{11}(t) \rightarrow a_{0} /\left|f^{\prime}(q)\right|$ as $t \rightarrow \infty$, we come to the required assertion.

Let us pass to consideration the case $a=0$. Using the finiteness of the second moment $f^{\prime \prime}(1)=: 2 b$ and from (30) after elementary transformations we find

$$
1-\frac{R(t ; s)}{R(t)} \sim \frac{s}{(1-s) b t+1}, \quad t \rightarrow \infty .
$$

In turn, according to (30) and (55), $R(t) / P_{11}(t) \sim a_{0} t$ as $t \rightarrow$ $\infty$. Then considering (84) and (87) we obtain

$$
\mathscr{M}(t ; s) \sim \frac{a_{0} s t}{(1-s) b t+1}, \quad t \longrightarrow \infty .
$$

The last relation proves (83).

Remark 12. Assertions of last two theorems along with the Lemma 3 in case $a \neq 0$ allow us to judge about asymptotic behavior of the sum $\sum_{j \in \mathbb{N}} \mu_{j}$. In the case $a<0$ it converges and diverges if $a \geq 0$. 
In fact when $a<0$ the integral (42) converges and according to Lemma $3 \mathscr{A}(s \uparrow 1)=0$. Hence, owing to (82) we find the sum

$$
\mathscr{M}(s \uparrow 1)=\sum_{j \in \mathbb{N}} \mu_{j}=\frac{a_{0}}{|a|}<\infty .
$$

In the case $a>0$ as established in Lemma 3 that $\mathscr{A}(s \uparrow$ 1) $=-\infty$. Therefore

$$
\sum_{j \in \mathbb{N}} \mu_{j}=+\infty
$$

Finally when $a=0$ relation (83) shows that

$$
\mathscr{M}(s) \sim \frac{a_{0}}{b} \cdot \frac{1}{1-s}, \quad s \uparrow 1 .
$$

Hence from the Hardy-Littlewood Tauberiam theorem it follows

$$
\lim _{n \rightarrow \infty} \frac{1}{n}\left[\mu_{1}+\mu_{2}+\cdots+\mu_{n}\right]=\frac{a_{0}}{b} .
$$

The last equality means that in the critical case $\sum_{j=1}^{n} \mu_{j}=O(n)$ as $n \rightarrow \infty$.

Now using the Lemma 7 from Theorems 4-6 we can establish the following assertions which give complete account on limit properties of transition function $P_{i j}(t)$.

Theorem 13. If $a<0$ and $\sum_{j \in \mathbb{N}} a_{j} j \ln j<\infty$ or $a>0$ then

$$
\beta^{-t} P_{i j}(t)=\frac{\mathscr{A}(0)}{\mathscr{M}(q)} i q^{i-1} \mu_{j}(1+o(1)), \quad t \longrightarrow \infty .
$$

Theorem 14. Let in critical MBP $Z(t)$ the second moment $f^{\prime \prime}(1)=: 2 b$ be finite. Then the following representation holds:

$$
t^{2} P_{i j}(t)=\frac{i \mu_{j}}{a_{0} b}\left(1+O\left(\frac{1}{t}\right)\right), \quad t \longrightarrow \infty .
$$

Further we will discuss the role of the set of $\left\{\mu_{j}, j \in \mathbb{N}\right\}$ as invariant measures for MBP. As it has been noticed above $\mu_{1}=1$ and $\mu_{j}<\infty$ for $j \in \mathbb{N}$ and $\mu_{j}>0$ for $j \in S$, where $S=\left\{j \in \mathbb{N}: P_{1 j}(t)>0, t \in \mathscr{T}\right\}$. It is clear $P_{00}(t)=1$. Then according to condition (58) $\mu_{0}^{*}=0$ for any invariant measure $\left\{\mu_{j}^{*}, j \in \mathbb{N}\right\}$. If $P_{10}(t)=0$ then equality (58) becomes

$$
\mu_{j}^{*}=\sum_{k=1}^{j} \mu_{k}^{*} P_{k j}(t) .
$$

If $P_{10}(t)>0$ then $P_{i 0}(t)>0$ and hence $\mu_{j}^{*}>0$.

Theorem 6 shows that in noncritical situation transition functions $P_{i j}(t)$ exponentially decrease to zero. The limit

$$
\lambda_{Z}=-\lim _{t \rightarrow \infty} \frac{\ln P_{i i}(t)}{t}
$$

that is independent on $i \in \mathbb{N}$ characterizes a decay of the state space of chain $Z(t)$. It is called the decay parameter of states of this chain. MBP is classified as $\lambda_{Z}$-transient if

$$
\int_{0}^{+\infty} e^{\lambda_{Z} t} P_{i i}(t) d t<\infty
$$

and $\lambda_{Z}$-recurrent otherwise. In this case invariant measure is called $\lambda_{Z}$-invariant. According to the general classification MBP is called $\lambda_{Z}$-positive if $\lim _{t \rightarrow \infty} e^{\lambda_{Z} t} P_{i i}(t)>0$ and $\lambda_{Z^{-}}$ null if this is zero; see [10]. Theorems 10 and 13 imply the following theorem.

Theorem 15. Let $a \neq 0$ and $\sum_{j \in \mathbb{N}} a_{j} j \ln j<\infty$ if $a<0$. Then

$$
\lambda_{Z}=\left|f^{\prime}(q)\right|
$$

and MBP is $\lambda_{Z}$-positive. The set of $\left\{\mu_{j}, j \in \mathbb{N}\right\}$ determined by the GF (82) is the unique (up to multiplicative constant) $\lambda_{Z^{-}}$ invariant measure.

In critical case the set $\left\{\mu_{i}\right\}$ directly enters to a role of invariant measure for the MBP. Indeed, in this case $\beta=1$ and as it has been proved in Theorem 8 , the following relation holds:

$$
\mu_{j}=\sum_{k \in \mathbb{N}} \mu_{k} P_{k j}(t), \quad j \in \mathbb{N} .
$$

Moreover according to (92), $\sum_{j \in \mathbb{N}} \mu_{j}=\infty$.

As shown in Theorems 13 and 14 hit probabilities of MBP to any states through the long interval time depend on the initial state. That is ergodic property is not carried out. Thereby we will seek the ergodic chain nearly MBP.

Recall

$$
T:=\inf \{t \in \mathscr{T}: Z(t)=0\}
$$

that is, extinction moment of the MBP. We see that following relations hold:

$$
\begin{gathered}
\mathrm{P}_{i}\{T=t\}=F^{i}(t ; 0)-F^{i}(\tau ; 0), \\
\mathrm{P}_{i}\{t<T<\infty, Z(t)=j\}=P_{i j}(t) \cdot q^{j}, \\
\mathrm{P}_{i}\{t<T<\infty\}=\sum_{j \in \mathbb{N}} P_{i j}(t) q^{j},
\end{gathered}
$$

here $t, \tau \in \mathscr{T}, 0<\tau<t$. In fact, since definition

$$
\mathrm{P}_{i}\{T=t\}=\mathrm{P}_{i}\{Z(t)=0, Z(\tau)>0\},
$$

for any $t, \tau \in \mathscr{T}$ and $0<\tau<t$, hence by the formula of full probability we come to (101). To receive (102) write

$$
\begin{aligned}
\mathrm{P}_{i}\{t & <T<\infty, Z(t)=j\} \\
& =\mathrm{P}\{t<T<\infty \mid Z(t)=j\} \cdot P_{i j}(t) .
\end{aligned}
$$

Since the probability of extinction of $j$ particles is $q^{j}$ then it follows (102). Finally the equality (103) is a direct consequence of (102):

$$
\begin{aligned}
\mathrm{P}_{i}\{t<T<\infty\} & =\sum_{j \in \mathbb{N}} \mathrm{P}_{i}\{Z(t)=j, t<T<\infty\} \\
& =\sum_{j \in \mathbb{N}} P_{i j}(t) q^{j} .
\end{aligned}
$$


Now put into consideration conditional transition function

$$
\widetilde{P}_{i j}(t):=\mathrm{P}_{i}\{Z(t)=j \mid t<T<\infty\}
$$

and define a corresponding GF

$$
\mathscr{V}_{i}(t ; s)=\sum_{j \in \mathbb{N}} \widetilde{P}_{i j}(t) s^{j}
$$

Theorem 16. If $a<0$ and $\sum_{j \in \mathbb{N}} a_{j} j \ln j<\infty$ or $a>0$, then limits

$$
\lim _{t \rightarrow \infty} \widetilde{P}_{i j}(t)=v_{j}, \quad j \in \mathbb{N},
$$

exist and these are determined by the GF:

$$
\mathscr{V}(s)=\frac{\mathscr{M}(q s)}{\mathscr{M}(q)},
$$

where the function $\mathscr{M}(s)$ is defined in (82).

Proof. By virtue of equalities (102) and (103) and using the Lemma 7 we have

$$
\begin{aligned}
\widetilde{P}_{i j}(t) & =\frac{P_{i}\{Z(t)=j, t<T<\infty\}}{P_{i}\{t<T<\infty\}} \\
& =\frac{\left(P_{i j}(t) / P_{11}(t)\right) \cdot q^{j}}{\sum_{k \in \mathbb{N}}\left(P_{i k}(t) / P_{11}(t)\right) q^{k}} \rightarrow \frac{\mu_{j} \cdot q^{j}}{\sum_{k \in \mathbb{N}} \mu_{k} q^{k}} \\
& =\frac{\mu_{j} q^{j}}{\mathscr{M}(q)}=: v_{j},
\end{aligned}
$$

as $t \rightarrow \infty$. Undoubtedly to the found limit corresponds to the GF (110).

Remark 17. Proved Theorem 16 is a generalization of wellknown results of Theorem A in which corresponding result is established in subcritical situation only. Indeed it is easy to see that the limit probability GF (22) is the proprietary case of the one (110).

Remark 18. The set $\left\{v_{j}\right\}$ presents a distribution of probabilities since setting $s=1$ in (110) and taking into account (82) it follows that $\mathscr{V}(1)=\sum_{j \in \mathbb{N}} v_{j}=1$. Moreover

$$
\sum_{j \in \mathbb{N}} j \widetilde{P}_{i j}(t) \longrightarrow \frac{q}{\mathscr{A}(0)}, \quad t \longrightarrow \infty,
$$

and this limit distribution has a finite mean $\mathscr{V}^{\prime}\left(\begin{array}{lll}s & 1\end{array}\right)=$ $q / \mathscr{A}(0)$.

In the critical situation $\mathrm{P}\{T<\infty\}=1$ and

$$
1-F^{i}(t ; s) \sim i R(t ; s),
$$

since $\sup _{0 \leq s<1} F(t ; s) \rightarrow 1$ as $t \rightarrow \infty$. Therefore seeing (84) we have

$$
\begin{aligned}
\mathscr{V}_{i}(t ; s) & =\sum_{j \in \mathbb{N}} \mathrm{P}_{i}\{Z(t)=j \mid T>t\} s^{j} \\
& \sim 1-\frac{R(t ; s)}{R(t)}=\frac{P_{11}(t)}{R(t)} \cdot \mathscr{M}(t ; s) .
\end{aligned}
$$

Hence it directly follows from Theorem 11 the following theorem.

Theorem 19. Let $a=0$. If $f^{\prime \prime}(1)=: 2 b<\infty$, then

$$
t \mathscr{V}_{i}(t ; s)=\frac{1}{b} \cdot \frac{s}{1-s}+\rho(t ; s)
$$

where $\sup _{0 \leq s<1}|\rho(t ; s)|=O(1 / t)$ as $t \rightarrow \infty$.

Now define the stochastic process $\widetilde{Z}(t)$ with the transition function $\left\{\widetilde{P}_{i j}(t)\right\}$. It is easy to be convinced that $\widetilde{Z}(t)$ represents MBP. Indeed probabilities $\widetilde{P}_{i j}(t)$ satisfy KolmogorovChapman equation (2) and have the branching property (3). According to last theorems properties of trajectories of $\widetilde{Z}(t)$ lose dependence on the initial state as $t \rightarrow \infty$.

In a noncritical situation under the condition of Theorem 16 for the MBP $\widetilde{Z}(t)$ there is (up to multiplicative constant) unique set of nonnegative numbers $\left\{v_{i}\right\}$ which are not all zero and we see the GF $\mathscr{V}(s)=\sum_{j \in \mathbb{N}} \nu_{j} s^{j}$ satisfies the invariance equation:

$$
\beta^{t} \cdot \mathscr{V}(s)=\mathscr{V}\left(\frac{F(t ; q s)}{q}\right)-\mathscr{V}\left(\frac{F(t ; 0)}{q}\right)
$$

Thus we have the following theorem.

Theorem 20. Let $a \neq 0$ and $\sum_{j \in \mathbb{N}} a_{j} j \ln j<\infty$ in case of $a<$ 0. Then

$$
P_{i j}(t)=\widetilde{P}_{i j}(t) \cdot \sum_{k \in \mathbb{N}} P_{i k}(t) q^{k-j},
$$

where transition functions $\widetilde{P}_{i j}(t)$ have an ergodicity property and their limits $v_{j}=\lim _{t \rightarrow \infty} \widetilde{P}_{i j}(t)$ present an invariant distribution for the Markov chain $\widetilde{Z}(t)$.

In the critical situation the following assertion holds, as a direct corollary of Theorem 19.

Theorem 21. If in critical situation $2 b:=f^{\prime \prime}(1)<\infty$, then

$$
t \widetilde{P}_{i j}(t) \longrightarrow \frac{1}{b}, \quad t \longrightarrow \infty .
$$

\section{Concluding Remarks}

We devote the paper to research of limit properties of MBP $Z(t), t \in \mathscr{T}$. Thus our focus has concentrated exclusively on the transition functions of this process. All our reasoning and results are based on the assertion of Lemma 2 (DA Basic Lemma) which in turn is the consequence of Lemma 1 . In noncritical case we strongly depend on the function $\mathscr{A}(s)$. Thereby we had to investigate properties of this function in detail. Therefore we managed to improve classical results on local properties of states of $Z(t)$. In fact, Theorems 4 and 5 refine corresponding results of the paper [11]. 
Monotone ratio Lemma 7 plays an important role in studying of ergodic property of chain $Z(t)$. The discrete analogue of this lemma in the Galton-Watson process (GWP) case can be found in the monograph [12, Chapter I.7]. Statements of Theorems 8 and 9 are also continuous analogues of corresponding results in the case of GWP. Forms of GF $\mathscr{M}(s)=$ $\lim _{t \rightarrow \infty} \mathscr{M}(t ; s)$ stated in Theorems 10 and 11 supplement our representation about properties of invariant measures of MBP. Theorems 13 and 14 play the same role for asymptotic properties of transition probabilities $P_{i j}(t)$. We are sure that statements of Theorems 10-14 are fair as well for the GWP situation and these will appear in our subsequent papers.

Theorems 16 and 19 assert that the Markov chain generated by transition function $\widetilde{P}_{i j}(t)$, has an ergodic property. And its limits $v_{j}=\lim _{t \rightarrow \infty} \widetilde{P}_{i j}(t)$ form an invariant measure. In GWP case the similar statement has been proved in the monograph [12, Chapter I.8]. Qua continuation of our discussion we note that this measure defines a new homogeneous Markov chain called the Q-process. The Q-process in discrete time case was introduced in [9, Chapter I.14] and in continuous time case it was considered by the author [13]. An investigation of properties of Markov Q-processes is our next research topic.

\section{Conflict of Interests}

The author declares that there is no conflict of interests regarding the publication of this paper.

\section{Acknowledgments}

The author would like to express his sincere thanks for the anonymous referee for his careful reading of the paper, helpful comments, and suggestions. The author is also grateful to Professor Anthony Pakes, who he has never met, but whose many papers inspired the author in his researches on Branching Processes and made writing this paper such a pleasure.

\section{References}

[1] A. N. Kolmogorov and N. A. Dmitriev, "Branching stochastic process," Reports of Academy of Sciences of USSR, vol. 56, pp. 307-315, 1947 (Russian).

[2] T. E. Harris, Theory of Branching Stochastic Process, Mir Publisher, Moscow, Russia, 1966, (Russian).

[3] B. A. Sevastyanov, Branching Processes, Nauka, Moscow, Russia, 1971, (Russian).

[4] B. A. Sevastyanov, "The theory of branching random processes," Uspekhi Matematicheskikh Nauk, vol. 6, no. 46, pp. 47-99, 1951 (Russian).

[5] C. R. Heathcote, E. Seneta, and D. Vere-Jones, "A refinement of two theorems in the theory of branching processes," Theory of Probability and Its Applications, vol. 12, no. 2, pp. 341-346, 1967.

[6] A. V. Nagaev and I. S. Badalbaev, "A refinement of certain theorems on branching random process," Litovskiy Matematicheskiy Sbornik, vol. 7, no. 1, pp. 129-136, 1967 (Russian).
[7] V. M. Zolotarev, "More exact statements of several theorems in the theory of branching processes," Theory of Probability and Its Applications, vol. 2, no. 2, pp. 256-266, 1957 (Russian).

[8] V. P. Chistyakov, "Local limit theorems in theory of branching random process," Theory of Probability and Its Applications, vol. 2, no. 3, pp. 341-346, 1957 (Russian).

[9] W. Anderson, Continuous-Time Markov Chains: An Applications-Oriented Approach, Springer, New York, NY, USA, 1991.

[10] J. Li, A. Chen, and A. G. Pakes, "Asymptotic properties of the Markov branching process with immigration," Journal of Theoretical Probability, vol. 25, no. 1, pp. 122-143, 2010.

[11] A. A. Imomov, "A differential analog of the main lemma of the theory of Markov branching processes and its applications," Ukrainian Mathematical Journal, vol. 57, no. 2, pp. 307-315, 2005.

[12] K. B. Athreya and P. E. Ney, Branching Processes, Springer, New York, NY, USA, 1972.

[13] A. A. Imomov, "On Markov analogue of Q-processes with continuous time," Theory of Probability and Mathematical Statistics, no. 84, pp. 57-64, 2012. 


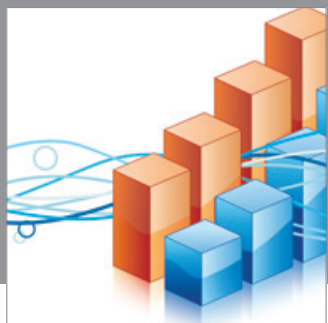

Advances in

Operations Research

mansans

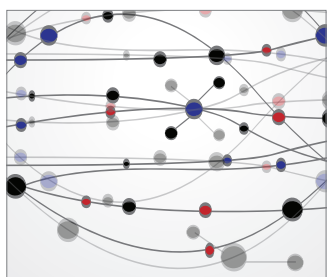

The Scientific World Journal
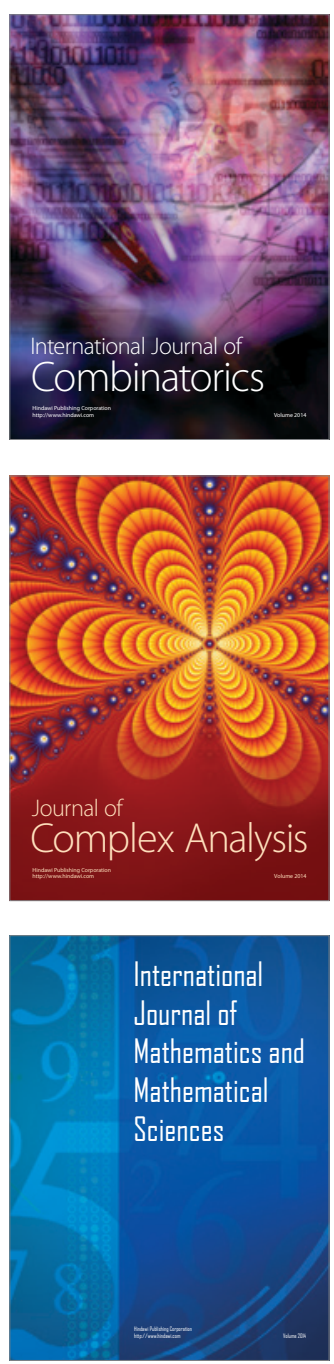
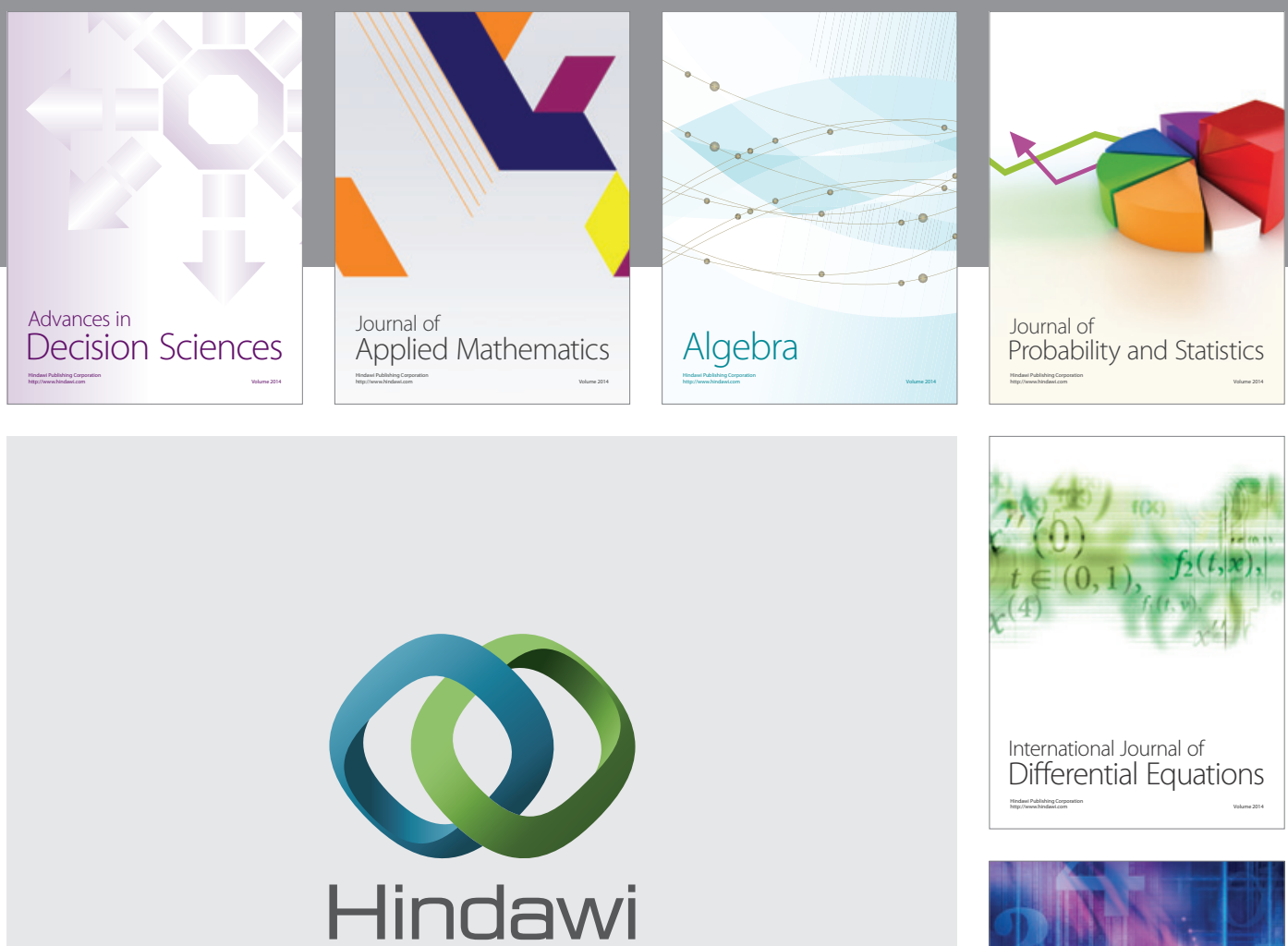

Submit your manuscripts at http://www.hindawi.com
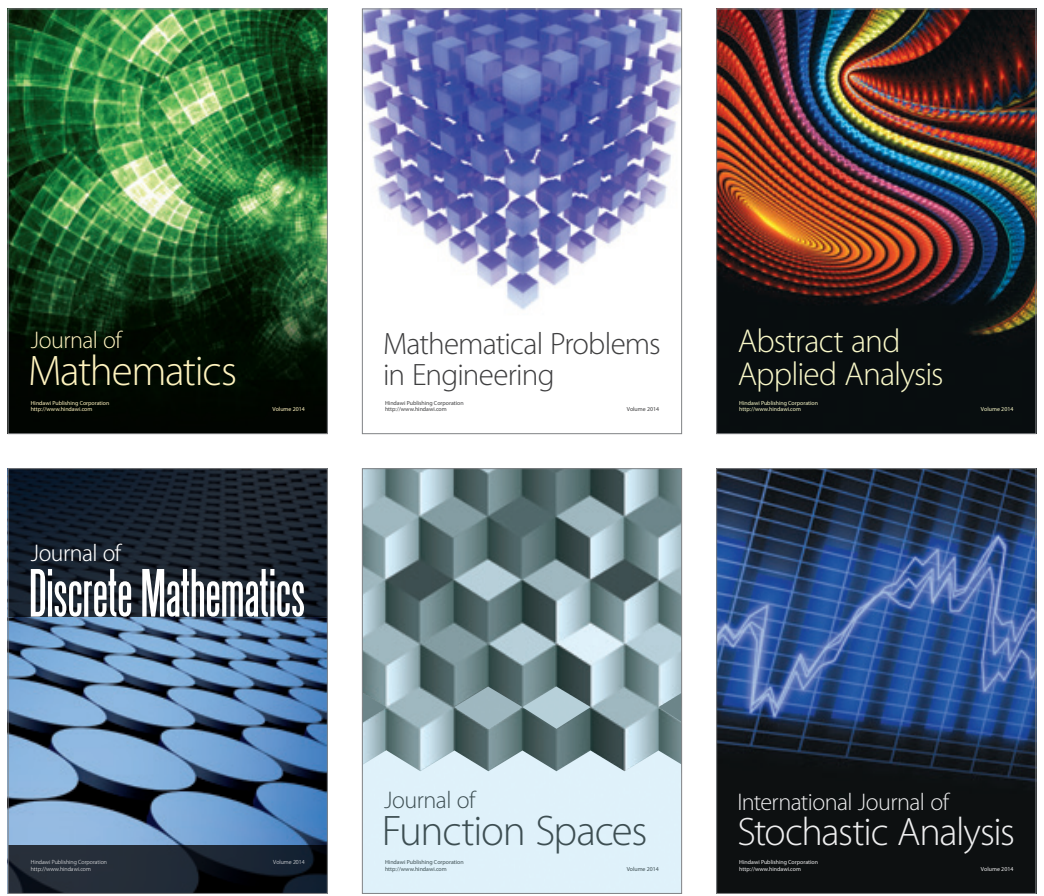

Journal of

Function Spaces

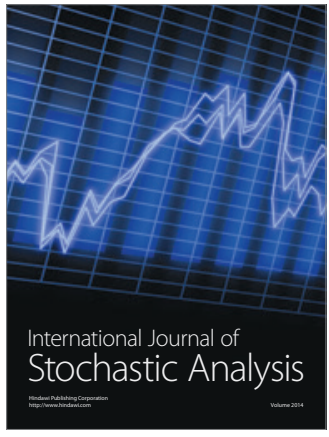

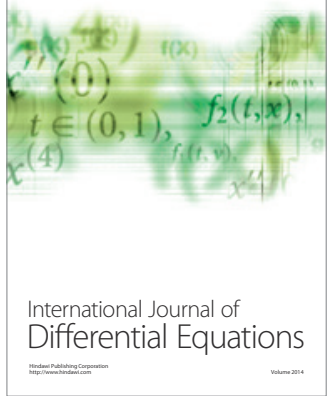
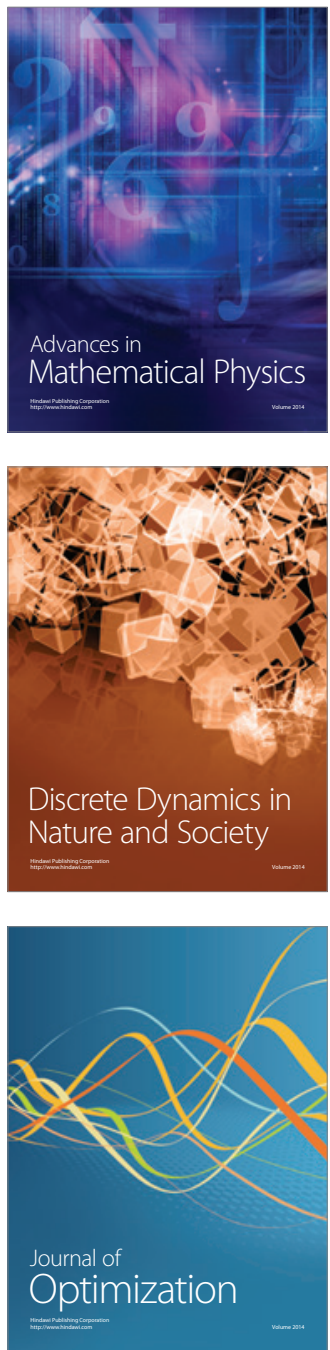